\title{
Introduction to the Intellectualization of Agrotechnology Management
}

\section{ISSN : 2688-836X}

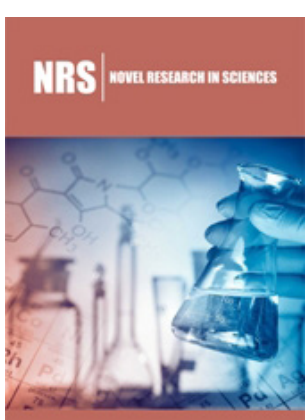

*Corresponding author: Mikhaylenko IM, Agrophysical Institute, St. Petersburg, Russia

Submission: 烸 August 01, 2019

Published: 㘹August 02, 2019

Volume 1 - Issue 4

How to cite this article: Mikhaylenko IM. Introduction to the Intellectualization of Agrotechnology Management. Nov Res Sci.1(4). NRS.000518.2019.

DOI: 10.31031/NRS.2019.1.000518

Copyright@ Mikhaylenko IM, This article is distributed under the terms of the Creative Commons Attribution 4.0 International License, which permits unrestricted use and redistribution provided that the original author and source are credited.

\section{Mikhaylenko IM*}

Agrophysical Institute, St. Petersburg, Russia

Opinion

The concept of digital economy was formed in the last decade of the last century. Nicholas Negroponte, an informatics specialist, founder of the Media Labs of the Massachusetts Institute of Technology (MIT) [1], first formulated the fundamental principles of the digital economy. Agriculture is on the verge of the Second Green Revolution. Experts believe that, thanks to precision farming technologies (PF) based on the Internet of Things, a surge in yields of such magnitude that humanity did not see even during the advent of tractors, the invention of herbicides and genetically modified seeds may follow. By its very nature, PF implies the solution of the problem of increasing the manageability of crop production by solving a set of tasks related to the management of agricultural technologies. All this set of tasks is divided into two groups, solved in different ways and means. The first group is the tasks of organizational management that are solved by management at various levels. For their implementation in modern conditions, decision support systems (DSS) are used. The second group is the direct management of agricultural technologies, which is implemented by automated control systems (ACS). Intellectualization of management in both groups depends on the degree of participation of the person himself, which in turn is due to the scientific and technical level of the DSS and ACS projects.

In the DSS and ACS with analytical blocks of control or decision-making, the modern theory of estimation and control of state parameters of controlled systems is implemented. Here, control decisions are made on the basis of procedures for minimizing optimality criteria, reflecting the accepted control goal. It is obvious that to minimize the optimality criteria in analytical DSS and ACS it is necessary to implement quite a lot of measurement and computational procedures. These include verification of measurement information, identification of multiparameter mathematical models with their subsequent adaptation in real time, estimation of parameters of the state of controlled objects and systems, according to which optimality criteria are evaluated, implementation of control decision-making algorithms themselves and development of control commands for robotic technological machines [2]. For their implementation requires highly qualified staff. It is difficult to provide in modern agro firms and farms.

This problem can be solved by switching to cloud computing technologies, when computer resources and capacities are provided to the user as an Internet service [3]. The capabilities of cloud technologies allow implementing the full range of procedures necessary for making management decisions in analytical DSS and ACS. However, this does not simplify the work of service personnel, who, in addition to performing the entire complex sequence of calculations to find optimal control solutions, must ensure effective data exchange with the cloud. The situation is greatly simplified in the transition to expert systems in which control decisions are made directly from the input information, bypassing complex multi-step computational procedures. The main information core of expert systems is the knowledge base (KB), which is the information basis for making management decisions. At the same time, it should be borne in mind that the principle of coding expert knowledge cannot be used to form a KB. After all, no matter how well qualified he does not have, optimal solutions are not available to him. 
It is possible to ensure high efficiency of expert systems if, instead of an expert, the software and hardware complexes of analytical DSS and ACS are used as an information source [2]. Through such complexes, a large number of optimal solutions are formed for various soil and climatic conditions for the cultivation of crops and a variety of initial situations. Each such case is an element of the knowledge base and is the optimal control solution for the given conditions, ensuring the maximization of the result in a controlled system. The formation of the KB optimal solutions for the given conditions is a serious foundation that provides a high scientific and technical level of the management process. Figure 1 shows the block diagram of the DSS system with training from the data center. Here in the data center, by repeatedly solving a control problem or making management decisions, a KB is formed supplemented by a control algorithm (management decision making). Through the public cloud, the KB together with the control algorithm is transferred to local DSS, where, according to the actually measured data on the state of the controlled system, the current remote sensing data and meteorological parameters, control decisions are made or commands are generated for robotic technological machines.

The transfer of knowledge bases and control algorithms to local control systems is accompanied by some loss of optimality, obtained by direct decision by analytical DSS or ACS. The main source of such losses is the difference in the parameters of mathematical models of the data processing center and local control systems. These differences are due to differences in the physical parameters of the soil, the relief features of the fields, and the various productive properties of the crop varieties. Therefore, the most important research tasks in the direction of the intellectualization of agrotechnology managements is the assessment of the loss of optimality of the decisions made when transferring KB from information processing centers to local DSS and ACS (Figure 1).

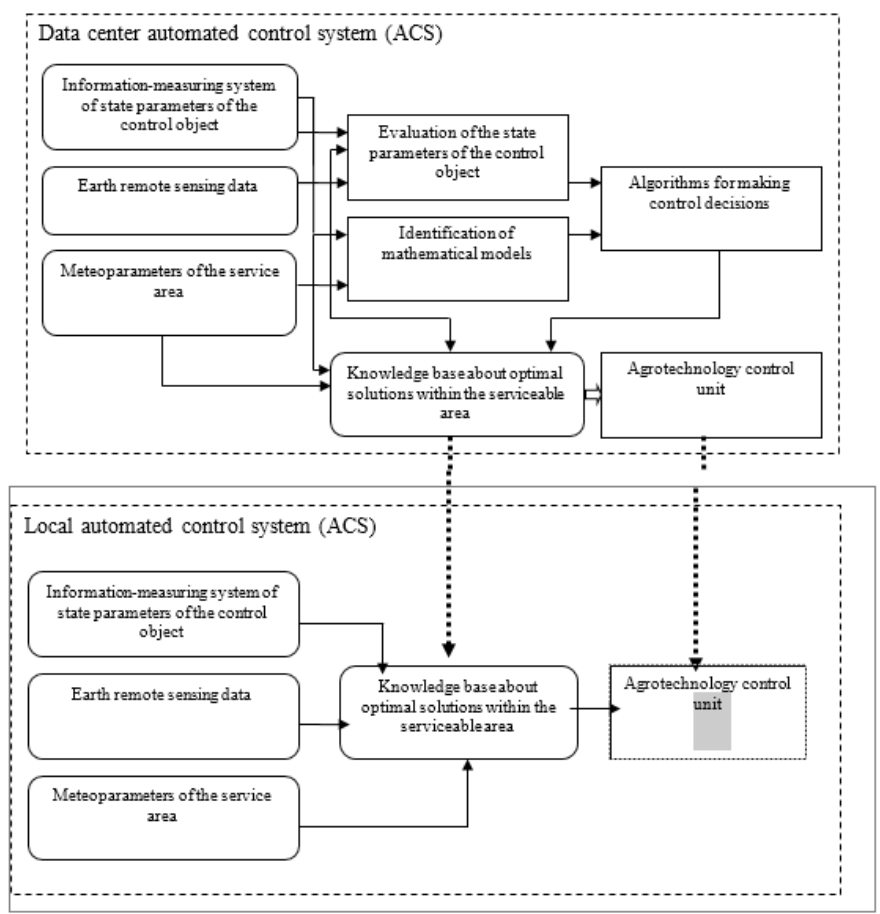

Figure 1: Block diagram of the automated process control system with training from the data center.

\section{References}

1. Neogronte N (1995) Vintage Books, New York.

2. Mikhaylenko IM (2017) Theoretical foundations and technical implementation of agrotechnology management. St. Petersburg State Technical University. p. 250.

3. Joseph M. Proceedings of the 1st ACM symposium on Cloud computing / Hellerstein.

For possible submissions Click below: 